\title{
Parâmetros lineares da marcha de crianças com paralisia cerebral do tipo espástica: estudo de caso
}

\author{
Linear parameters of the march of children with \\ cerebral palsy of the spastic type: case study
}

\author{
Paula Hortência dos Santos Magalhães ${ }^{1}$ (1) \\ Joane Gabriela Souza Oliveira ${ }^{2}$ (1) \\ Vitor Sotero dos Santos 3 (1) \\ Menilde Araújo Silva Bião4 (1)
}

19Autora para correspondência. Faculdade Estácio de Feira de Santana (Feira de Santana). Bahia, Brasil. dra.hortenciamagalhaes@gmail.com ${ }^{2,4}$ Faculdade Estácio de Feira de Santana (Feira de Santana). Bahia, Brasil. jgabriela.fisio@gmail.com, menildearaujo@hotmail.com ${ }^{3}$ Universidade Federal da Bahia (Salvador), Centro Universitário Ruy Barbosa (Salvador). Bahia, Brasil. vitorsotero@gmail.com

RESUMO | INTRODUÇÃO: A paralisia cerebral (PC) ocorre devido lesão no sistema nervoso central, resultando em comprometimento funcional e alterações na marcha. OBJETIVO: Verificar e descrever as variáveis lineares da marcha de crianças com paralisia cerebral do tipo espástica. MATERIAIS E MÉTODOS: Participaram do estudo três crianças com idade entre seis e sete anos com paralisia cerebral do tipo espástica, GMFCS nível I e II, onde foi realizada a identificação do desempenho da função motora grossa por meio do Sistema de Classificação da Função Motora Grossa (GMFSC) com grau 3 e 4 de espasticidade em membros inferiores segundo a Escala Modificada de Ashworth (MAS). Para registro dos parâmetros lineares e organização dos dados foram utilizados o software livre CVMob e o método de análise de movimento MED Movement Element Decomposition. RESULTADOS: Os valores encontrados para velocidade foram $(0,64 ; 0,58 ; 0,96 \mathrm{~m} / \mathrm{s}) \mathrm{com}$ primento do passo $(0,34 ; 0,36 ; 0,36 \mathrm{~m})$ cadência $(127 ; 118 ; 130$ p/min). CONCLUSÃO: Foi verificada a diminuição das variáveis lineares da marcha para velocidade, comprimento do passo e cadência nas três crianças estudas. Apesar da dificuldade em obter amostra maior, os dados sugerem a necessidade de maior controle das variáveis da marcha de crianças com Paralisia cerebral do tipo espástica.

PALAVRAS-CHAVE: Paralisia cerebral. Marcha. Distúrbios motores. Fenômenos biomecânicos.

\begin{abstract}
INTRODUCTION: Cerebral palsy (CP) occurs due to injury to the central nervous system resulting in functional impairment and gait changes. OBJECTIVE: To verify and describe the linear gait variables of children with static cerebral palsy. MATERIALS AND METHODS: Three children aged between six and seven years with spastic cerebral palsy, GMFCS level I and II participated in the study, where the performance of gross motor function was identified by means of the Thick Motor Function Classification System (GMFSC) with grades 3 and 4 of spasticity in the lower limbs according to the Modified Ashworth Scale (MAS). To record the linear parameters and data organization, the cvmob free software and the motion movement - Movement Element Decomposition method were used. RESULTS: The values found for speed were $(0.64 ; 0.58$; $0.96 \mathrm{~m} / \mathrm{s})$ step length $(0.34 ; 0.36 ; 0.36 \mathrm{~m})$ cadence $(127 ; 118$; $130 \mathrm{p} / \mathrm{min}$ ). CONCLUSION: There was a reduction in linear gait variables for speed, length and cadence in the three children studied. Despite the difficulty in obtaining larger samples, the data suggest the need for greater control of gait variables in children with spastic cerebral palsy.
\end{abstract}

KEYWORDS: Cerebral palsy. Gait. Motor disturbances. Biomechanical phenomena. 


\section{Introdução}

As lesões neurológicas que ocorrem na infância quando o encéfalo se encontra em fase de desenvolvimento e maturação, ocasionam alterações no sistema nervoso que podem resultar na encefalopatia crônica não progressiva também conhecida como paralisia cerebral $(P C)$, que se caracteriza pela presença de distúrbios estacionários, provocando desordens no desenvolvimento motor da criança, limitando profundamente a sua funcionalidade ${ }^{1}$.

Estima-se que sua incidência nos países desenvolvidos é de 2,0 a 2,5 por 1000 nascidos vivos, já nos países que se encontram em desenvolvimento é de $7 / 1000$ nascidos vivos ${ }^{2}$. No Brasil são aproximadamente 30000 a 40000 novos casos por ano, em $70 \%$ a $80 \%$ dos casos a paralisia cerebral ocorre no período pré-natal em decorrência de diversos fatores, dentre eles as malformações congênitas, infecções durante o período da gravidez, hipoxemia, traumas abdominais, prematuridade e baixo peso ${ }^{1,2}$.

As manifestações clinicas da paralisia cerebral variam de acordo com a área cerebral acometida (cerebelo, córtex cerebral, núcleos da base), sua etiologia é classificada como atáxica, extrapiramidal, espástica e mista. As características topográficas se dividem em diparesia, quadriparesia e a hemiparesia ${ }^{1}$. A paralisia cerebral do tipo espástica corresponde a $70 \%$ dos casos, afetando o sistema musculoesquelético provocando aumento do tônus muscular com padrão patológico na reciprocidade da inibição de músculos antagonistas, gerando uma coativação excessiva dos músculos, reforçando o comprometimento funcional ${ }^{1,3}$.

A marcha é a função mais acometida na paralisia cerebral espástica, ela permite a transferência de um lugar para outro. O padrão normal da marcha consiste em ciclos que são a passada quando o calcanhar que tocou o solo volta a toca-lo e o passo que é a distância entre os dois calcanhares ${ }^{4}$. As fases que compõe são: contato inicial, a fase de apoio, (inicial, médio, final,) fases de balanço, (inicial, médio e final), para prosseguir com um novo ciclo ${ }^{5}$.

Devido à presença da espasticidade comprometer a função muscular, o excesso de flexão plantar, conhecido como pé equinovaro, altera o posicionamento do membro e modifica o ciclo da marcha ${ }^{6}$. Isto provoca alterações de equilíbrio que, para garantir a sua estabilidade e a realização da marcha, observa-se a realização de movimentos compensatórios para manter um padrão de locomoção seguro¹. Essas modificações apesar de serem a melhor solução encontrada para realizar a locomoção, representam um risco para o surgimento de encurtamento, contraturas e possíveis deformidades que alteram e prejudicam na dinâmica da marcha ${ }^{6}$.

Considerando que a marcha é uma habilidade motora importante para o deslocamento e a independência funcional, envolvendo descargas e transferências de peso nos membros, é importante identificar as alterações presentes para definir as alterações existentes. Dessa forma, o objetivo deste estudo consistiu em verificar e descrever as variáveis lineares da marcha de crianças com paralisia cerebral do tipo espástica.

\section{Metodologia}

Foi realizada uma pesquisa do tipo quase-experimental, também conhecido como experimentos não aleatórios, onde o grupo de pesquisa em questão é analisado sem e com algum tipo de intervenção.

Este estudo foi realizado na Associação de Pais de Amigos dos Excepcionais (APAE) de Feira de SantanaBahia, localizada na rua São Cosme e Damião, Bairro Santa Mônica. Por sua vez, trata-se de uma associação civil, filantrópica, de caráter assistencial, educacional, cultural, de saúde, estudo e pesquisa, sem fins lucrativos, que presta atendimento educacional a crianças e adolescentes com deficiência.

Participaram do estudo crianças com paralisia cerebral do tipo espástica com idade entre 6 e 7 anos, classificada nos níveis I e II do Sistema de Classificação da Função Motora Grossa (GMFCS) com capacidade de atender a comandos verbais simples como "ficar de pé, andar, parar, dar a volta".

Foram excluídas da pesquisa aquelas com deficiência múltipla do tipo, auditiva, visual e intelectual; discrepância de membros inferiores; displasia do quadril; aquelas que foram submetidas a procedimentos cirúrgicos nos últimos 12 meses e/ou aplicação de toxina botulínica e fenol nos últimos seis meses antes da coleta dos dados, bem como as que apresentaram graus 3 e 4 de espasticidade em membros inferiores, segundo a Escala Modificada de Ashworth. Foi solicitado autorização dos pais ou responsáveis 
das crianças mediante assinatura do Termo de Consentimento Livre e Esclarecido.

Para avaliar a função motora foi utilizada a escala Gross Motor Function Classification System (GMFCS), que classifica em níveis a função motora grossa da criança com ênfase no movimento de sentar e caminhar por meio de cinco níveis motores presentes em cada uma das quatro faixas etárias (0 a 2 anos, 2 a 4 anos, 4 a 6 anos e 6 a 12 anos). Os níveis são classificados de forma ordinal em: Nível 1: caminha sem restrições, possui limitação nas atividades motoras mais complexas. Nível 2: Caminha sem restrições, apresenta dificuldade no ambiente e na comunidade Nível 3: caminha com recurso auxiliar, limitação no ambiente. Nível 4: possuem limitação na mobilidade, necessitam ser transportadas, usam recurso para mobilidade. Nível 5: Mobilidade acometida severamente, necessita de recursos de Tecnologia Assistiva ${ }^{7}$.

O grau de espasticidade das crianças, foi avaliado com a Escala Modificada de Ashworth (MAS), que se trata de uma escala de 6 pontos e objetiva a precisão relacionada à avaliação do tônus, quantificando a resistência passiva da musculatura. Os pontos da MAS são: 0: Tônus normal, 1: Pouco aumento do tônus muscular, com resistência no final da ADM, quando as partes que foram afetadas, são movimentadas em flexão e extensão, 1+: Pouco aumento no tônus, referido pelo apreender, com mínima resistência através de menos da metade, da amplitude de movimento, 2: Destacável aumento do tônus muscular, por meio de uma amplitude de movimento maior, porém as partes afetadas exercem movimentos com facilidade, 3: Relevante aumento do tônus, com movimentos passivos prejudicados. 4: A parte afetada apresenta rigidez nos movimentos de flexão ou extensão ${ }^{8}$.

A primeira etapa da análise da marcha correspondeu à adaptação dos participantes por 3 metros em linha reta, no local destinado à coleta de marcha em velocidade auto selecionada. Finalizada a adaptação ao local de coleta, os marcadores reflexivos foram fixados nos seguintes pontos anatômicos: coxa esquerda (trocanter maior), joelho esquerdo (côndilo lateral), tornozelo esquerdo (maléolo lateral) e no $5^{\circ}$ dedo do pé esquerdo, por ser o membro acometido nas crianças selecionadas.

Para a captação dos dados cinemáticos, foi selecionado um local amplo, com boa iluminação e superfície plana sem irregularidades, foram utilizados uma câmera
Nikon p510, um tripé para fixação da câmera e adesivos desenvolvidos pelas pesquisadoras para marcação dos pontos anatômicos da criança. Foi solicitado que as mesmas executassem a marcha, com 6 passos, para que 3 fossem captados para análise.

A análise da marcha consistiu na realização de passos no ambiente de avaliação, onde os participantes realizaram o mesmo percurso da sessão de adaptação em 7 tentativas, até que 6 passos fossem capturados integralmente. Os participantes realizaram a análise de pés descalços. O presente estudo seguiu os parâmetros de normalidade da marcha e as variáveis espaço-temporais de velocidade, cadência e comprimento de passo propostos por Dusing, Thorpe ${ }^{9}$.

Para a realização desta pesquisa, foi feita a análise dos riscos que poderiam ser levados ao sujeito ou responsável, como: desconforto, devido ao acompanhamento dos profissionais à criança para a coleta de dados, constrangimento aos pais e/ou responsáveis em responder ao questionário, expectativa e/ou ansiedade e queda durante a marcha.

Para a minimização destes riscos, foi imprescindível a presença dos pais e/ou responsáveis, o local utilizado para a análise foi devidamente iluminado, com piso seco e de superfície plana, sem objetos ou desníveis que poderiam oferecer o maior risco de queda, e a equipe esteve todo o tempo presente para proporcionar segurança a criança e os responsáveis. Os benefícios desta pesquisa incluíram: a acessibilidade, inclusão, destacar a importância do acompanhamento fisioterapêutico e de uma avaliação biomecânica precisa com relação ao uso de um dispositivo devidamente adequado para reduzir os riscos de quedas e de deformidades.

A análise dos resultados foi realizada segundo estatística descritiva (frequência absoluta/relativa) com a finalidade de identificar as características gerais e específicas da amostra estudada. Os dados cinemáticos foram registrados no software livre CVMob, e utilizado o método de análise de movimento MED Movement Element Decomposition.

Para a preservação dos aspectos éticos da pesquisa, o estudo foi devidamente aprovado pelo Comitê de Ética do Centro Universitário Estácio da Bahia Estácio FIB de acordo com a resolução do Conselho Nacional de Saúde- CNS 466/2012, sob o número de CAAE 07025219.1.0000.0041. 


\section{Resultados}

As características sociodemográficas são apresentadas na tabela 1, das 03 crianças incluídas no estudo, 02 são do sexo masculino e 01 do sexo feminino, a idade média dos participantes foi de $\pm 6,66$, com idade mínima 06 anos e máxima 07 anos.

Tabela 1. Características sociodemográficas da população estudada, 2019

\begin{tabular}{lccc}
\hline CARACTERÍ́STICAS & C1 & C2 & C3 \\
\hline Idade & 6 & 7 & 7 \\
Sexo & $\mathrm{F}$ & $\mathrm{M}$ & $\mathrm{M}$ \\
Raça & $\mathrm{B}$ & $\mathrm{N}$ & $\mathrm{N}$ \\
Estudante & $\mathrm{SIM}$ & $\mathrm{SIM}$ & SIM
\end{tabular}

Fonte: características sociodemográficas das crianças selecionadas para o estudo. F- Feminino, M- masculino, B- branco, $\mathrm{N}$ - negro. Elaborado pelas autoras,2019.

Quanto à função motora grossa, de acordo com a GMFCS, duas crianças foram classificadas em grau I, por conseguirem realizar a marcha de forma independente e sem restrições. A outra criança foi classificada com grau II, apesar de conseguir deambular sem uso de dispositivo que auxilia na marcha, possui dificuldades para realizar atividades mais complexas, apresentando queda com frequência no ambiente doméstico e comunitário. Sobre o diagnóstico topográfico das crianças participantes do estudo, duas possuíam hemiparesia e uma tetraparesia. De acordo com a Escala de Ashworth o maior grau de espasticidade encontrado nas crianças avaliadas foi 02 , presente nos músculos extensores de joelho e plantiflexores, conforme apresentado na Tabela 2.

Ao avaliar a marcha das crianças, todas apresentavam rotação interna de quadril, padrão flexor em joelho durante a fase de apoio inicial e médio, fazendo a extensão apenas na fase de apoio final e inversão do pé, apenas uma criança realizava o contato inicial com o antepé, os outros realizavam com o calcanhar, entretanto, o movimento de dorsiflexão não era feito de forma completa. Todos os pacientes apresentavam leve/moderada diminuição no equilíbrio estático e dinâmico, realizando movimentos como alargamento do passo e anteriorização do tronco para se manterem equilibrados.

Comparando os valores da marcha das crianças com paralisia cerebral com os valores normais estabelecidos por Dusing e Thorpe, observou-se que a cadência, comprimento dos passos e a velocidade tiveram uma tendência a ter valores menores nas crianças com paralisia cerebral avaliadas neste estudo. Essas diferenças estão representadas na Tabela 3. 


\begin{tabular}{|c|c|}
\hline Características & Sujeitos da pesquisa $(n=3)$ \\
\hline \multicolumn{2}{|l|}{ Funcionalidade (\%) } \\
\hline GMFCS nível I & $2(66,66 \%)$ \\
\hline GMFCS nível II & $1(33,33 \%)$ \\
\hline \multicolumn{2}{|l|}{ Topografia (\%) } \\
\hline Hemiparesia & $2(66,67 \%)$ \\
\hline Tetraparesia & $1(33,33 \%)$ \\
\hline \multicolumn{2}{|l|}{ Grau de espasticidade - n (\%) } \\
\hline \multicolumn{2}{|l|}{ Extensores do quadril (D/E) } \\
\hline Grau 0 & 2 (D/E) \\
\hline Grau 1+ & $1(\mathrm{E})$ \\
\hline \multicolumn{2}{|l|}{ Flexores do quadril (D/E) } \\
\hline Grau 0 & 2 (D/E) \\
\hline Grau 1+ & 1 (D/E) \\
\hline \multicolumn{2}{|l|}{ Abdutores de quadril (D/E) } \\
\hline Grau 0 & 3 (D/E) \\
\hline \multicolumn{2}{|l|}{ Adutores do quadril (D/E) } \\
\hline Grau 0 & 2 (D/E) \\
\hline Grau 2 & $1(\mathrm{D})$ \\
\hline \multicolumn{2}{|l|}{ Flexores do joelho (D/E) } \\
\hline Grau 0 & 1 (D/E) \\
\hline Grau 1 & $2(1-E ; 1-D / E)$ \\
\hline \multicolumn{2}{|l|}{ Extensor de joelho (D/E) } \\
\hline Grau 0 & 1 (D/E) \\
\hline Grau 1 & $1(E)$ \\
\hline Grau 2 & 1 (D/E) \\
\hline \multicolumn{2}{|l|}{ Plantiflexores (D/E) } \\
\hline Grau 0 & $1(\mathrm{D} / \mathrm{E})$ \\
\hline Grau 1+ & 1 (D/E) \\
\hline Grau 2 & $1(\mathrm{E})$ \\
\hline \multicolumn{2}{|l|}{ Dorsiflexores (D/E) } \\
\hline Grau 0 & 2 (D/E) \\
\hline Grau 1+ & $1(\mathrm{D} / \mathrm{E})$ \\
\hline
\end{tabular}

Fonte: Fonte: Nível l: caminha sem restrições, possui limitação em atividades motoras mais complexas. Nível 2: Caminha sem restrições, apresenta dificuldade no ambiente e na comunidade. (D- direito/ E- esquerdo). Elaborado pelas autoras, 2019.

Tabela 3. Distribuição das variáveis espaço-tempo comparados com os valores que definem o padrão de normalidade

\begin{tabular}{lcccc}
\hline \multicolumn{1}{c}{ Padrão da marcha } & Média \pm normal & C1 & C2 & C3 \\
\hline Velocidade $(\mathrm{m} / \mathrm{s})$ & 1,25 & 0,64 & 0,58 & 0,96 \\
Comprimento do passo $(\mathrm{m})$ & 0,58 & 0,34 & 0,36 & 0,36 \\
Cadência $(\mathrm{p} / \mathrm{min})$ & 137,8 & 127 & 118 & 130
\end{tabular}

Fonte: média normal (Dusing; Thorpe ${ }^{9}$ M/s- Metros por segundo; p/min- passos por minuto; Comparação com os valores da normalidade. Elaborado pelas autoras, 2019. 


\section{Discussão}

O presente estudo apresentou a análise dos parâmetros lineares da marcha de três crianças com paralisia cerebral do tipo espástica, comparando com os valores normais para marcha, apresentados na literatura. Os valores estabelecidos por Dursing; Thorpe ${ }^{9}$, mostram que a média da velocidade, comprimento do passo e cadência apresentam valores de 1,25, 0,58 e 137,8 respectivamente, os valores destes parâmentos foram menores nas crianças avaliadas. As crianças 1 (C1), 2 (C2) e 3 (C3), apresentaram valores de 0,64, 0,58, e 0,96 $\mathrm{m} / \mathrm{s}$ para velocidade, 0,34 , e 0,36 para comprimento do passo e 127, 118 e 130 para cadência.

Os valores da velocidade podem variar de $0,62,0,64$ e $0,71 \mathrm{~m} / \mathrm{s}$ em crianças com funcionalidade I e II na escala GMFCS, esses valores podem se apresentar menores com o maior grau de comprometido da PC. A marcha em crianças com paralisia cerebral, quando comparada com a marcha de crianças que não possuem limitações motoras e com desenvolvimento típico, apresenta diferentes valores nos parâmetros lineares, os valores podem se apresentar inferiores nas crianças com PC, e as alterações motoras podem contribuir para esta diminuição ${ }^{10}$.

As principais alterações biomecânicas presentes encontradas nas crianças deste estudo foram: rotação interna de quadril, flexão de joelho e diminuição na dorsiflexão do tornozelo. Para Muller e Valentini ${ }^{11}$ essas alterações provocam mudanças no posicionamento e na amplitude de movimento, implicando em uma diminuição na velocidade, comprimento do passo e cadência.

Na paralisia cerebral, o movimento artrocinemático de rolar, no tornozelo está ausente ou diminuído, devido alterações na posição do joelho e tornozelo na fase de contato inicial da marcha, em decorrência da espasticidade, provocando alterações na velocidade, cadência, comprimento do passo e distribuição de peso, gerando maior gasto energético ${ }^{12}$. Quando o contato inicial é feito pela parte frontal do pé, há uma diminuição no recrutamento das fibras do tibial anterior, comprometendo a extensão do joelho e quadril, que permanecem semi-fletidos durante a marcha implicando o seu desenvolvimento típico ${ }^{6}$.

Esta condição é definida devido a presença do pé equino, provocado pela espasticidade nos músculos do tríceps sural e fraqueza no tibial anterior que reflete no posicionamento do membro, modificando o ciclo da marcha ${ }^{11}$. A espasticidade também faz com que a musculatura não cresça proporcionalmente ao crescimento dos ossos, levando a contraturas e diminuição no tamanho das fibras, resultando em alteração no comprimento dos passos e da passada. A postura adotada em flexão plantar no tornozelo e flexão de joelho, é usada como mecanismo de compensação devido à instabilidade na marcha ${ }^{13,11}$.

Fernandes et al. ${ }^{3}$ afirma que crianças com paralisia cerebral (PC) possuem dificuldades funcionais relevantes por disporem de um padrão musculoesquelético primitivo, uma diminuição de controle proprioceptivo e motor, e pela espasticidade, que por sua vez, impulsionam uma ativação neuromuscular simultânea do músculo antagonista quando há o estímulo voluntário alterando os ciclos e as fases da marcha.

As alterações dinâmicas presentes na marcha são corrigidas por órteses. A utilização de órtese em crianças com PC é essencial para propiciar estabilidade, evitar contraturas, deformidades e encurtamentos musculares, oferecendo assim, auxílio funcional e autonomia ao indivíduo ${ }^{13,1}$.

Os resultados de um estudo comparativo de crianças com PC, com e sem o uso da órtese realizado por Bride et al. ${ }^{12}$, encontrou diferenças significativas apenas no aumento do comprimento dos passos e passada com o uso da órtese, já os resultados de Cury et al. ${ }^{4}$ mostraram que o uso de órteses suropodálicas propiciou alterações benéficas nos parâmetros qualitativos e no desempenho motor grosso de crianças com PC, influenciando principalmente no posicionamento do pé na fase de contato inicial, melhorando a distribuição de peso durante a fase de apoio, reduzindo o equinismo dinâmico observado.

Desta forma a órtese tornozelo e pé ou suropodálica tem indicações por possuírem benefícios relevantes na marcha de crianças com PC, pois controla o excesso de plantiflexão e auxilia na dorsiflexão, possibilitando mudanças nos padrões lineares e na diminuição do gasto energético. No entanto, para sua prescrição o profissional deve estar atento ao seu objetivo e ao tipo de órtese a ser indicada levando em consideração aspectos patológicos, e as alterações encontradas mediante as análises ${ }^{12,1}$. 
Saad et al. ${ }^{14}$ relata que a marcha é uma sequência de movimentos acelerados e de difícil compreensão, com grande complexidade na avaliação sendo que sua análise é de fundamental importância em estudos e tratamento de patologias que estão prontamente envolvidas com o sistema locomotor. Para Sizinio et al. ${ }^{15}$ a marcha normal é um pré-requisito para analisar e avaliar marchas patológicas afirmando também que a análise tem sido vista como boa ferramenta de pesquisa, mesmo enfrentando adversidades acerca da escassez de laboratório, pouco investimento em exames e na capacitação de profissionais.

Os resultados obtidos no presente estudo identificam uma tendência a alterações nos valores da marcha de crianças com paralisia cerebral, que estão diretamente relacionadas às condições de tônus muscular e suas limitações motoras. O conhecimento da marcha típica é um fator que determina na identificação de alterações na marcha patológica, dessa forma a análise da marcha utilizando a ferramenta de baixo custo, pode ter implicações positivas para avaliação e na determinação do plano de tratamento, auxiliando também na prescrição correta dos dispositivos como órtese para assegurar o bem-estar e a independência funcional do indivíduo.

Devido à limitação no número de participantes deste estudo, não foi possível aplicar nenhum teste estatístico, entretanto, os valores espaço-temporais encontrados na avaliação da marcha de crianças com PC do tipo espástica são alterados diante das condições clinicas e motoras presentes. Dados semelhantes encontrados em outros estudos apostam nessas diferenças, relatando o benefício do uso de órteses como ferramenta imprescindível na correção do posicionamento do membro inferior. Os resultados deste estudo podem auxiliar no processo de avaliação e prescrição de órteses, bem como contribuir para futuras pesquisas que tratem sobre este assunto.

\section{Conclusão}

Os resultados encontrados para parâmetros lineares demostraram que houve diminuição na velocidade, cadência e comprimento do passo na marcha das crianças com paralisia cerebral, quando comparadas com valores normais estabelecidos na literatura.
Neste estudo os valores podem ser esperados para essa população em decorrência do posicionamento incorreto do membro, provocado pelas alterações no tônus muscular provocada pela presença da espasticidade. Apesar da amostra pequena, há indícios de uma maior necessidade de controle das variáveis da marcha de crianças com Paralisia Cerebral do tipo espástica.

\section{Contribuições dos autores}

Magalhães PHS, Oliveira JGS participaram da coleta e análise dos dados e redação do artigo. Sotero $\mathrm{V}$ orientou e supervisionou quando ao uso do Cvmob e do Med, avaliação dos vídeos e organização dos dados. Bião MAS supervisionou a pesquisa, participou da análise dos dados e da redação do artigo.

\section{Conflitos de interesses}

Nenhum conflito financeiro, legal ou político envolvendo terceiros (governo, empresas e fundações privadas, etc.) foi declarado para nenhum aspecto do trabalho submetido (incluindo, mas não se limitando a subvenções e financiamentos, participação em conselho consultivo, desenho de estudo, preparação de manuscrito, análise estatística, etc.).

\section{Referências}

1. Oliveira ACT, Ballarino $H$, Monteiro MR, Pinto NA, Pires ELSR. Análise da visão de forma de colocação de órtese tornozelo-pé pelos pais ou cuidadores de paciente com paralisia cerebral. Rev Bras Clin Med, 2010;8(6):490-4.

2. Zanini G, Cemin NF, Peralles SN. Paralisia Cerebral: causas e prevalências. Fisioter Mov. 2009;22(3):375-381.

3. Fernandes TL, Lima KB, Silva PRS, Oshiro MS, Paula A. Avaliação da marcha em paciente com paralisia cerebral submetido à estimulação elétrica dos compartimentos anterior e lateral da perna. Acta Fisiátr. 2011,18(1):42-44.

4. Cury VCR, Mancini MC, Melo AP, Fonseca ST, Sampaio RF, Tirado MGA. Efeitos do uso de órtese na mobilidade funcional de crianças com paralisia cerebral. Rev Bras Fisioter. 2006;10(1):6774. doi: 10.1590/S1413-35552006000100009

5. Mafra NR. Análise de Imagem na Avaliação Clínica da Marcha Humana [monografia]. Porto: Universidade do Porto; 2012.

6. Mélo TR. Análise Cinemática da Marcha de crianças com Diparesia Espástica em plano inclinado [dissertação]. Curitiba: Universidade Federal do Paraná; 2011. 
7. Capucho PY, Carnier SADC, Souza P, Castro DC, Finocchio. AP, Oliveira DM et al. Paralisia cerebral - membros inferiores: reabilitação. Acta Fisiatr. 2012;19(2):114-22. doi: 10.5935/0104$\underline{7795.20120018}$

8. Teixeira LF, Olney SJ, Brauwer B. Mecanismos e medidas de espasticidade. Rev Fisioter Univ São Paulo. 1998;5(1):4-19. doi: 10.1590/fpusp.v5i1.76781

9. Dusing SC, Thorpe DE. A normative sample of temporal and spatial gait parameters in children using the GAITRite ${ }^{\circledR}$ electronic walkway. Gait Posture. 2007;25(1):135-9. doi: 10.1016/j. gaitpost.2006.06.003

10. Martinello M, Medeiros DL, Piucco EC, Ries LGK. Parâmetros cinemáticos da marcha de criança com paralisia cerebral: Comparação entre diferentes formas de apoio. Cad Ter Ocup. 2014;22(1):137-143. doi: 10.4322/cto.2014.015
11. Muller AB, Valentini NC. Análise Cinesiológica do pé Equinovaro na Criança com Paralisia Cerebral Espástica. R Bras Ci Saúde. 2016;20(3):253-258. doi: 10.4034/RBCS.2016.20.03.12

12. Bridi D, Cavião IC, Schmitt VM, Saccani R, Bonetti LV, Cechetti F et al. Análise da marcha de crianças com paralisia cerebral com e sem uso de órteses de tornozelo e pé. Sci Med. 2018;28(2). doi: $10.15448 / 1980-6108.2018 .2 .29390$

13. Carvalho JA. Órtese: um recurso Terapêutico Complementar. 2.ed. São Paulo: Manole; 2013.

14. Saad M, Battistella LR, Masiero D. Técnicas de Análise da Marcha. Acta Fisiátrica. 1996;3(2):23-26.

15. Hebert SK. Ortopedia e Traumatologia: princípios e prática. 5.ed. Porto Alegre: Artmed; 2016. 